Revista Brasil. Bot., V.28, n.4, p.785-795, out.-dez. 2005

\title{
Biologia reprodutiva de Psychotria suterella Muell. Arg. (Rubiaceae) e a abordagem de escalas ecológicas para a fenologia de floração e frutificação
}

\author{
LUCIANO E. LOPES ${ }^{1}$ e SILVANA BUZATO ${ }^{1,2}$
}

(recebido: 3 de fevereiro de 2005; aceito: 3 de novembro de 2005)

\begin{abstract}
Reproductive biology of Psychotria suterella (Rubiaceae) and the approach of ecological scales for flowering and fruiting phenology). Foraging behavior of pollinators and seed dispersers is related to resource heterogeneity in the environment, making it important to know the spatio-temporal variation of resources in order to understand the mutualistic relationships established between plants and animals. Psychotria suterella flowered from January to April, months with highest annual rainfall, and exhibited high synchronism among the populations in the landscape studied. The flowering pattern found on the category of individuals corresponds to that one found in populations and landscape categories. Fructification occurred all year long, with greater availability of mature fruits in May and December; and synchronism was lower in this phenophase than in flowering. Only intermorph pollinations produced fruits and seeds, indicating that this plant species relies on pollen vectors for sexual reproduction. Since there is no evidence of pollen limitation, we consider that differences in fructification among populations could be related to abiotic factors. The overlap between flowering pattern of Psychotria suterella and Bombus brasiliensis foraging activicty, the fact that B. brasiliensis is the most important pollinator of this plant species, as well as the importance of $P$. suterella for this bee diet, suggest that selective pressures could be acting between these species, underlying this mutualistic interaction.
\end{abstract}

Key words - ecological scales, floral traits, mutualism, pollination, reproductive phenology

RESUMO - (Biologia reprodutiva de Psychotria suterella (Rubiaceae) e a abordagem de escalas ecológicas para a fenologia de floração e frutificação). O comportamento de forrageio de polinizadores e dispersores de sementes é influenciado pela heterogeneidade de recursos no ambiente, sendo importante conhecer a variação espaço-temporal dos recursos a fim de compreender as relações mutualistas estabelecidas entre plantas e animais. Na paisagem estudada, a floração de Psychotria suterella ocorreu de janeiro a abril, meses de maior pluviosidade no ano, e apresentou alta sincronia entre as populações. O padrão fenológico de floração encontrado na categoria de indivíduo se assemelhou ao registrado para as categorias de população e paisagem. A frutificação ocorreu durante todo o ano, com maior disponibilidade de frutos maduros em maio e dezembro, havendo menor sincronia entre as populações e entre os indivíduos nesta fenofase do que na de floração. Apenas cruzamentos inter-morfos produziram frutos e sementes, indicando que esta espécie vegetal depende de vetores de pólen para reprodução sexuada. Uma vez que não há indícios de limitação polínica nas populações estudadas, consideramos que as diferenças na frutificação entre as populações poderiam estar associadas a fatores abióticos. A sobreposição entre o padrão de floração anual de Psychotria suterella e a atividade de forrageio de Bombus brasiliensis, o fato de B. brasiliensis ser o polinizador mais importante desta espécie vegetal, bem como a importância de $P$. suterella na dieta desta abelha, sugerem que pressões seletivas entre as duas espécies podem condicionar o resultado desta interação mutualista.

Palavras-chave - atributos florais, escalas ecológicas, fenologia, mutualismo, polinização

\section{Introdução}

Para a maioria das espécies animais e, em particular, para polinizadores e dispersores, a disponibilidade de recursos está irregularmente distribuída nos hábitats, sendo que o comportamento de forrageio destes animais pode ser influenciado pela heterogeneidade de recursos

\footnotetext{
1. Universidade de São Paulo, Instituto de Biociências, Departamento de Ecologia, Rua do Matão, travessa 14, 321, 05508-900 São Paulo, SP, Brasil

2._Autor para correspondência: sbuzato@usp.br
}

no ambiente (Rathcke 1992, Kunin \& Iwasa 1996). A heterogeneidade nos padrões fenológicos é reconhecidamente determinada por fatores tais como fotoperíodo, temperatura e pluviosidade (Rathcke \& Lacey 1985), e alteração de hábitat pode resultar em mudanças nas condições abióticas relacionadas aos fluxos de radiação, ventos e água (Saunders et al. 1991, Laurance et al. 2001), ocasionando diferenças na intensidade da floração (Fuchs et al. 2003). O momento, duração e intensidade da florada em espécies vegetais determinam as distâncias que os polinizadores terão que percorrer para obter alimento, influenciando a migração destes ao longo de gradientes temporais de recursos 
(Grant \& Grant 1967, Aker 1982, Tschapka 2004). Apesar da manutenção de polinizadores na paisagem estar relacionada às variações espaço-temporais na distribuição de recursos florais, questionamentos referentes a este assunto abordando diversas escalas ecológicas são raros (Bronstein 1995).

Nos sub-bosques das florestas tropicais, a família Rubiaceae é representada por grande número de espécies (Gentry \& Emmons 1987), exercendo grande influência na estrutura da vegetação. Várias de suas espécies são fontes de recursos para animais que se alimentam de pólen, néctar e frutos (Poulin et al. 1999, Castro \& Oliveira 2002, Lopes 2002, Melo et al. 2003), sendo componentes importantes para o funcionamento destas florestas. Tais particularidades fazem com que a família Rubiaceae seja relativamente bem estudada nos ecossistemas tropicais. Rubiaceae destaca-se também por apresentar mais gêneros heterostílicos do que todas as outras famílias em conjunto, sendo objeto de vários estudos evolutivos (Ganders 1979). A distilia é o tipo de heterostilia mais comumente encontrado na família, sendo adequado para estudos de dispersão de pólen (Feinsinger \& Busby 1987, Stone 1995, Lau \& Bosque 2003). Caracteriza-se morfologicamente pela existência de indivíduos do morfo brevistilo e longistilo, em cujas flores as anteras se posicionam acima ou abaixo do estigma, respectivamente (Barrett \& Richards 1990, Pailler \& Thompson 1997). É esperado que a reciprocidade na altura dos elementos florais de cada um dos níveis favoreça a dispersão direcional de grãos de pólen das anteras de um morfo para os estigmas do outro (Ganders 1979, Stone 1995, Barrett et al. 1998). Além das características morfológicas, a síndrome heterostílica apresenta mecanismo de incompatibilidade nos cruzamentos entre indivíduos do mesmo morfo que pode ser total ou parcial (Bawa \& Beach 1983, Barrett \& Richards 1990).

No Brasil, estudos da biologia reprodutiva de Rubiaceae tendem a abranger simultaneamente vários aspectos tais como fenologia, morfologia floral, sistema reprodutivo e interação com visitantes florais (Passos \& Sazima 1995, Machado \& Loiola 2000, Castro \& Oliveira 2001, 2002, Coelho \& Barbosa 2003), sendo que alguns estudos enfocam a fenologia (Almeida \& Alves 2000, Morellato et al. 2000, San Martin-Gajardo \& Morellato 2003) ou a frugivoria (Melo et al. 2003).

O objetivo deste estudo é caracterizar o sistema composto por Psychotria suterella Muell. Arg. quanto à morfologia floral e sistema reprodutivo. Além disto, a realização de observações fenólogicas em 12 populações possibilitou a avaliação dos eventos de floração e frutificação em diversas escalas ecológicas que contemplam indivíduos, populações e paisagem.

\section{Material e métodos}

Sistema de estudo - Psychotria suterella Muell. Arg. (Rubiaceae) possui hábito arbustivo a arbóreo, podendo alcançar até $6 \mathrm{~m}$ de altura (Carvalho et al. 2000). Nas áreas de estudo, há variações amplas na densidade populacional (21 a 1.250 ind.ha $^{-1}$, Lopes 2002), sendo esta uma das espécies mais abundantes no sub-bosque. As flores são tubulares, brancas e a antese dura cerca de $30 \mathrm{~h}$. Os principais polinizadores são abelhas do gênero Bombus, porém outras espécies de abelhas, borboletas e beija-flores visitam freqüentemente as flores (Lopes 2002).

Área de estudo - O presente estudo foi desenvolvido em oito fragmentos florestais pertencentes ao domínio da Mata Atlântica, nos municípios de Cotia e Ibiúna, SP $\left(23^{\circ} 35^{\prime} \mathrm{S}\right.$ $23^{\circ} 50^{\prime} \mathrm{S}$ e $46^{\circ} 45^{\prime} \mathrm{W}-47^{\circ} 15^{\prime} \mathrm{W}$ ) bem como em quatro

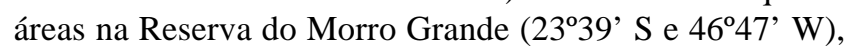
que possui 10.000 ha (Uezu et al. 2005). Neste estudo, foram denominados indivíduos, plantas de $P$. suterella com altura superior a $1 \mathrm{~m}$ e que distavam mais de $30 \mathrm{~cm}$ de outra planta da mesma espécie. Foi definido como população o conjunto de indivíduos de $P$. suterella em uma determinada área de estudo. A distância mínima entre duas populações foi de 492 $\mathrm{m}$, e a distância máxima foi de $11.044 \mathrm{~m}$. O conjunto de populações espacialmente distribuídas nos municípios de Cotia e Ibiúna, SP, permitiu a avaliação dos parâmetros fenológicos na escala de paisagem. A vegetação original da região é classificada como Floresta Tropical Chuvosa Baixo Montana (Oliveira Filho \& Fontes 2000). O clima da região, segundo a classificação de Köppen (1948) é Cwa, temperado e chuvoso, com pluviosidade mensal de $200-260 \mathrm{~mm}$ no verão e $60 \mathrm{~mm}$ no inverno e média das temperaturas mensais máximas no verão de $27^{\circ} \mathrm{C}$ e mínimas no inverno de $11^{\circ} \mathrm{C}$ (Sabesp 1997). Dados pluviométricos apresentados nas figuras 1 e 2 foram fornecidos pela ETA Alto Cotia, Sabesp. As áreas de estudo apresentam altitudes que variam de $850-1.100 \mathrm{~m}$. O processo de fragmentação na região foi intenso entre $1910 \mathrm{e}$ 1930, devido à exploração madeireira, sendo parte da vegetação atual oriunda de regeneração (Seabra 1971, Dean 1996, Uezu et al. 2005).

Fenologia - Foram sorteados 15 indivíduos de cada morfo para acompanhamento da fenologia de floração e frutificação dentro de uma área de $7.425 \mathrm{~m}^{2} \mathrm{em}$ cada uma das 12 populações. Neste trabalho são apresentados os valores médios e desvios padrão das intensidades de fenofases mensurados para 335 indivíduos de $P$. suterella, visto que alguns indivíduos marcados morreram durante o período de amostragem. De janeiro de 2001 a fevereiro de 2002, em amostragens fenológicas periódicas, foi registrada a ocorrência das fenofases de floração (emissão de flores) e frutificação (frutos maduros e imaturos) desses 
indivíduos. A intensidade de cada fenofase foi avaliada de acordo com a escala de Fournier (1974), a saber: $0=$ ausência da fenofase; 1 = presença da fenofase com magnitude entre $1 \%$ e $25 \% ; 2=$ presença da fenofase entre $26 \%$ e $50 \% ; 3=$ presença da fenofase entre $51 \%$ e $75 \%$ e $4=$ presença da fenofase entre $76 \%$ e $100 \%$. Considerando a intensidade de cada fenofase, foi calculada a porcentagem de Fournier pela seguinte fórmula: $F a=\left(\sum_{i=1}^{n} F i / 4 \times n\right) \times 100$; onde: $F a=$ Índice de Fournier de determinada fenofase na área $a$; $n=$ número de indivíduos observados; $F i=$ Índice de Fournier registrado no indivíduo $i$ (Fournier 1974). Além disso foi calculada a porcentagem de indivíduos que apresentava uma determinada fenofase, utilizando o dado de presença ou ausência da fenofase. A porcentagem de indivíduos mostra o período em que uma determinada fenofase ocorreu na maioria dos indivíduos de $P$. suterella, permitindo avaliar sincronia intra-específica. A porcentagem de Fournier é a variável a partir da qual os picos fenológicos podem ser evidenciados com maior precisão e refinamento, representando melhor o comportamento fenológico da espécie (Bencke \& Morellato 2002, San Martin-Gajardo \& Morellato 2003). Em trabalhos de fenologia, a apresentação gráfica dos resultados é comumente utilizada na análise (Newstrom et al. 1994, Bencke \& Morellato 2002, San Martin-Gajardo \& Morellato 2003), permitindo a avaliação concomitante de vários parâmetros fenológicos tais como duração e amplitude das fenofases. Dentre os vários estimadores de sincronia de eventos fenológicos (Augspurger 1983, Rathcke \& Lacey 1985, Marquis 1988, Gómez 1993, Bolmgren 1998), o desvio padrão foi escolhido para verificar a sincronia de floração e frutificação entre as populações bem como entre os indivíduos de uma população, dada a possibilidade dele ser expresso graficamente com os demais parâmetros fenológicos analisados. A fim de exemplificar o padrão fenológico em escalas ecológicas de população e indivíduo foi sorteada uma população de $P$. suterella dentre as 12 estudadas. A análise da variação fenológica nestas escalas a partir de uma das populações estudadas justifica-se pela alta sincronia dos eventos em $P$. suterella.

Morfologia floral - A caracterização morfológica das flores de $P$. suterella foi realizada a partir de quatro flores de oito indivíduos de cada morfo. As medidas obtidas foram: altura das anteras (da base do filete à extremidade superior da antera) e altura dos estigmas (da base do estilete à bifurcação do estigma) (Richards \& Koptur 1993), bem como comprimento interno do tubo da corola e diâmetro da corola (Buzato et al. 1994, Castro \& Oliveira 2002). Para comparações da morfologia floral entre morfos foi utilizado ANOVA com desenho hierárquico ("nested") (Zar 1999). As variáveis altura do estigma, comprimento e diâmetro da corola foram analisadas considerando flores agrupadas ("nested") em indivíduos e indivíduos em morfos. Na análise das alturas das anteras, estas foram agrupadas em flores. Para esta análise foram sorteadas quatro anteras por flor. Os componentes da variância $(\mathrm{CV})$ bem como o coeficiente de determinação $\left(\varpi^{2}\right)$ foram estimados de acordo com Graham \& Edwards (2001). O desenho experimental hierárquico permite levar em consideração a variação individual na morfologia floral. Os índices de reciprocidade referentes aos níveis superiores e inferiores de elementos florais foram obtidos a partir da expressão: (altura das anteras - altura do estigma recíproco)/(altura das anteras + altura do estigma recíproco) (Richards \& Koptur 1993). Os coeficientes de variação da altura das anteras e dos estigmas foram calculados a partir das alturas médias dessas estruturas em cada indivíduo, representando as variações entre indivíduos. Como o coeficiente de variação é um valor relativo, permite agrupar os indivíduos de ambos os morfos para comparar a variação em suas estruturas reprodutivas masculinas e femininas.

Sistema reprodutivo - A fim de determinar a expressão do sistema de incompatibilidade heteromórfico em $P$. suterella, caracterizando a ocorrência de incompatibilidade total ou parcial, foram realizados tratamentos de polinização manual: Autopolinização - estigma recebeu pólen da própria flor; Polinização intramorfos (cruzamento ilegítimo) - estigma recebeu pólen de outro indivíduo do mesmo morfo; Polinização intermorfos (cruzamento legítimo) - estigma recebeu pólen de indivíduo de morfo diferente (Ree 1997). Para todos os tratamentos, botões em pré-antese foram ensacados para evitar contato das flores com os visitantes florais. Além disto, flores foram deixadas sob condições naturais, com livre acesso aos visitantes florais. Todas as flores tratadas foram posteriormente ensacadas para acompanhamento da formação de frutos.

Frutificação - Foram ensacadas 511 flores de ambos os morfos de $P$. suterella em 10 populações estudadas para o acompanhamento da produção e tempo de maturação de frutos.

As estatísticas descritivas apresentadas neste estudo referem-se à média \pm desvio padrão.

\section{Resultados}

Padrões fenológicos de floração e frutificação - A floração de Psychotria suterella Muell. Arg., para as doze populações estudadas, ocorreu de janeiro a abril, sendo o pico de florada registrado em fevereiro (figura 1A). Verificamos pelos pequenos desvios padrão na porcentagem de indivíduos em flor que a sincronia entre as populações é alta (figura 1B). A frutificação ocorreu durante todo o ano, havendo dois momentos (maio e dezembro) de maior disponibilidade de frutos maduros (figura 1C). Os altos valores de desvios padrão indicam menor sincronia entre as populações nesta fenofase (figura 1D). Houve menor variação mensal entre as populações estudadas quanto à intensidade de floração que frutificação, sendo que a floração tende a apresentar sua maior intensidade associada aos períodos de maior precipitação (figura 1). Os padrões fenológicos de 
floração e frutificação encontrados para $P$. suterella na paisagem (figura 1) não diferem daqueles registrados para os da população e dos indivíduos tanto no que diz respeito à porcentagem de Fournier (figura $2 \mathrm{~A}, \mathrm{C}$ ) como para porcentagem de indivíduos (figura $2 \mathrm{~B}, \mathrm{D}$ ). $\mathrm{O}$ acompanhamento da frutificação, a partir das flores ensacadas, indicou que o desenvolvimento dos frutos é iniciado imediatamente após a florada, e que estes permanecem imaturos por pelo menos sete meses (figura 3). O tempo médio de maturação dos frutos foi de 12 meses, período no qual ocorreu a maturação de $50 \%$ dos frutos ensacados, sendo que $20 \%$ deles levaram mais de 13 meses para amadurecer (figura 3). A frutificação de $P$. suterella sob condições naturais variou de $6,9 \%$ a $55,2 \%$ nas 10 populações estudadas, com média e desvio padrão de $28 \% \pm 15,1$.

Morfologia floral - Dentre os atributos morfológicos florais analisados, somente a posição entre anteras e estigma da flor permitiu a identificação de dois morfos florais (brevistilo e longistilo) entre os indivíduos de $P$. suterella, não sendo registradas diferenças entre os morfos quanto ao comprimento e ao diâmetro do tubo da corola (figura 4, tabela 1). A altura das anteras e dos estigmas foi explicada, respectivamente, em $67,05 \%$ e $73,75 \%$ pelo fator morfo (tabela 2 ). Independente dos morfos, o coeficiente de variação na altura das anteras foi igual ao coeficiente de variação na altura dos estigmas, sendo respectivamente
A

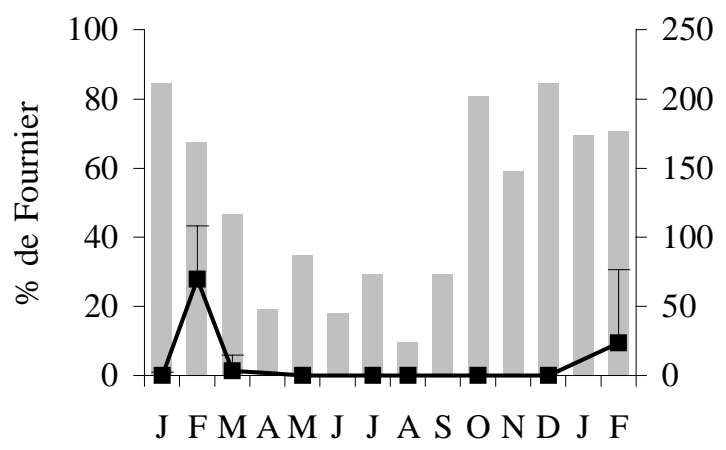

Meses

C

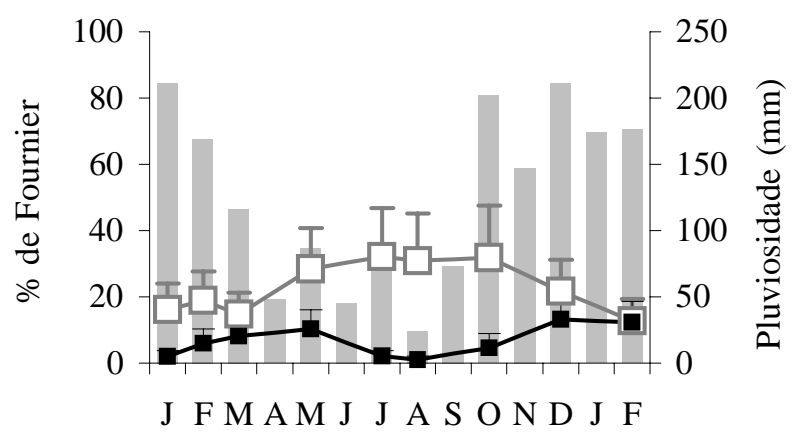

Meses

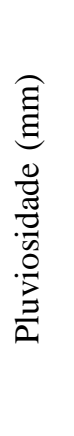

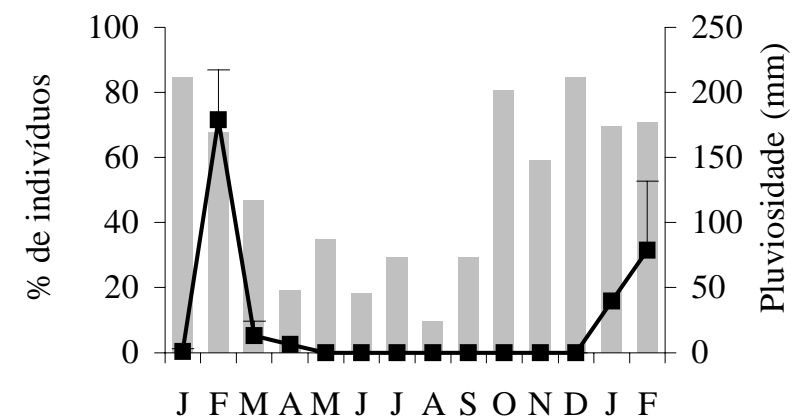

Meses

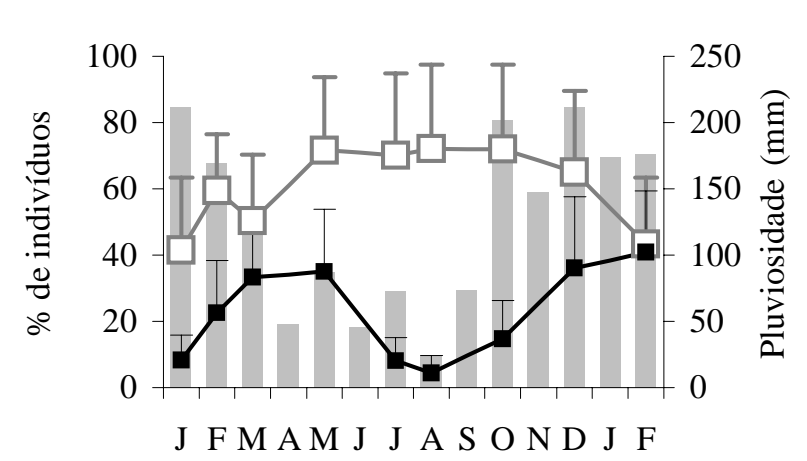

Meses

Figura 1. Fenologia de floração (A, B) e frutificação (C, D) em 12 populações de Psychotria suterella nos municípios de Cotia e Ibiúna, SP. Média e desvio padrão do Índice de Fournier das populações e do percentual de indivíduos na fenofase. Quadrados cheios representam flores (A, B) ou frutos maduros (C, D) e quadrados vazios representam frutos verdes (C, D). Pluviosidade no período - barras.

Figure 1. Flowering phenology (A, B) and fruiting phenology (C, D) for 12 populations of Psychotria suterella in Cotia and Ibiúna county, SP. Mean and standard deviations values for the Fournier index of the populations and for the percentage of individuals in each phenophase. Black squares are flowers (A, B) or mature fruits $(\mathrm{C}, \mathrm{D})$ and white squares are green fruits (C, D). Rainfall in gray bars. 
de $19,47 \%$ e $19,66 \%$. A diferença absoluta de altura entre antera e estigma foi explicada em $40,14 \%$ pelo fator flores em indivíduos. Este mesmo fator foi responsável por $58,70 \%$ da variação no comprimento da corola. Diferenças entre indivíduos de um mesmo morfo respondem por $87,19 \%$ da variação encontrada no diâmetro da corola (tabela 2). Houve sobreposição entre morfos na altura das anteras bem como na altura dos estigmas (figura 4), sendo o valor absoluto da diferença entre as alturas de anteras e estigmas maior no morfo brevistilo (tabela 1). Os índices de reciprocidade entre anteras e estigmas foram 0,005 para o nível superior e 0,036 para o nível inferior $(\mathrm{N}=36)$.
Sistema reprodutivo - Não houve formação de frutos e sementes em flores de $P$. suterella sob os tratamentos de autopolinização e polinização cruzada intra-morfos (tabela 3).

\section{Discussão}

Padrões fenológicos de floração e frutificação - Devido à labilidade fenológica na floração entre as espécies da família Rubiaceae, há consenso de que sua fenologia não sofre restrições filogenéticas (Wright 1991, San MartinGajardo \& Morellato 2003). Sendo assim, os padrões fenológicos de suas espécies podem ter resultado de pressões seletivas bióticas ou abióticas ocorridas no

A

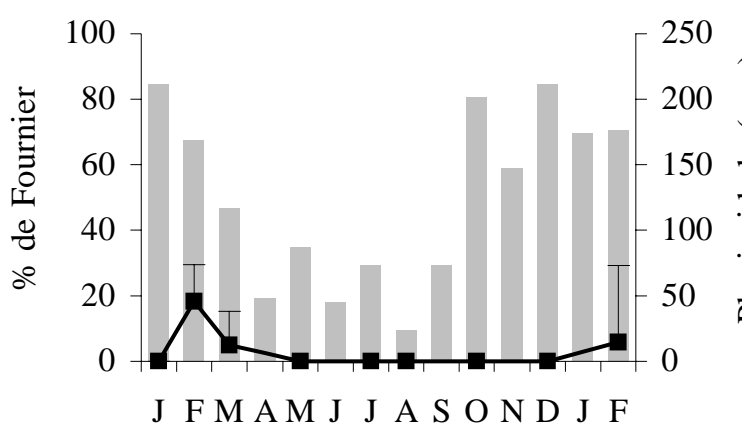

Meses

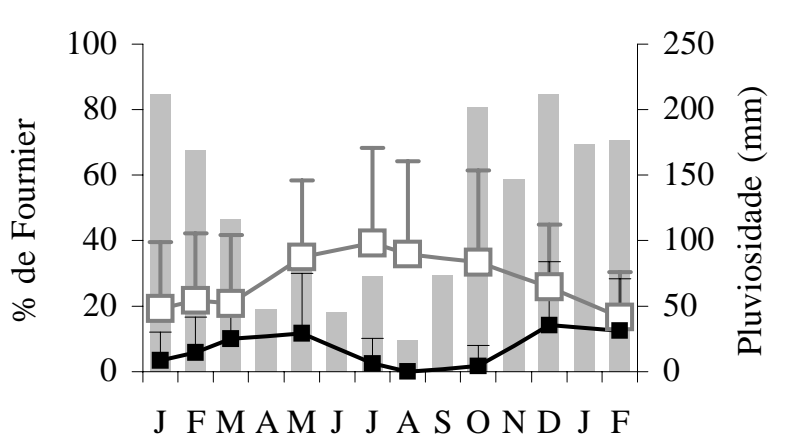

Meses

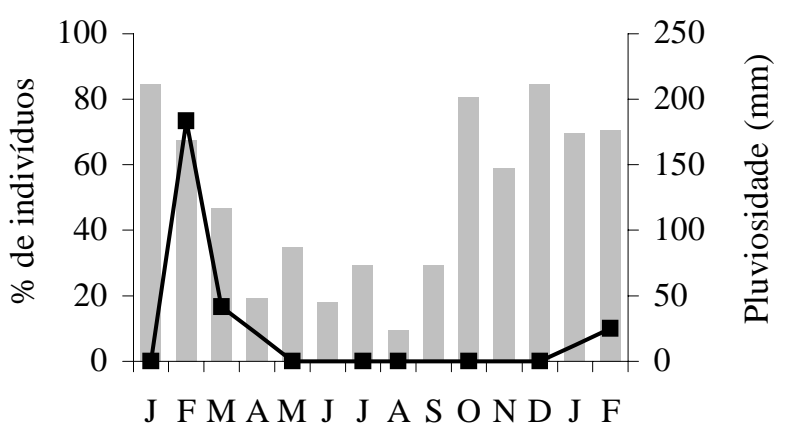

Meses

$\mathrm{C}$

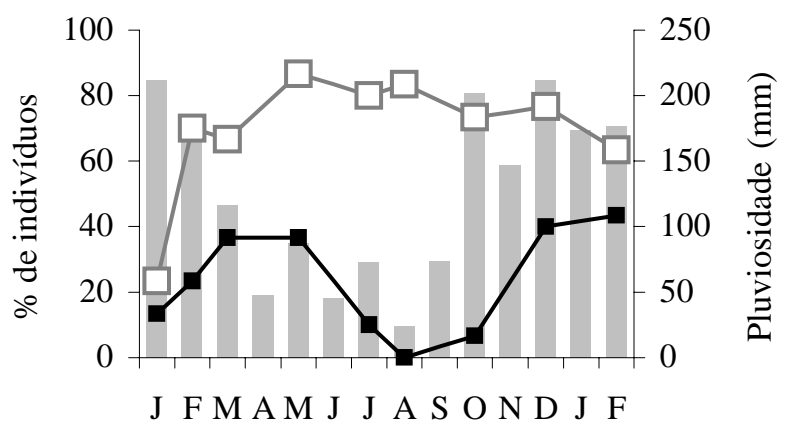

Meses

Figura 2. Fenologia de floração (A, B) e frutificação (C, D) de uma população de Psychotria suterella localizada no

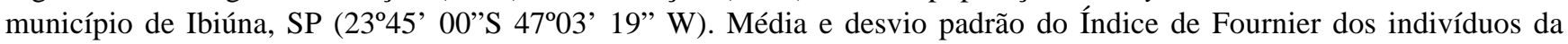
população e percentual de indivíduos na fenofase. Quadrados cheios representam flores (A, B) ou frutos maduros $(C, D)$ e quadrados vazios representam frutos verdes $(\mathrm{C}, \mathrm{D})$. Pluviosidade no período - barras.

Figure 2. Flowering phenology (A, B) and fruiting phenology (C, D) for one population of Psychotria suterella in Ibiúna county, SP (234' $00^{\circ}$ 'S $47^{\circ} 03^{\prime} 19^{\prime \prime}$ W). Mean and standard deviations values for the Fournier index of the populations and for the percentage of individuals in each phenophase. Black squares are flowers (A, B) or mature fruits (C, D) and white squares are green fruits (C, D). Rainfall in gray bars. 
passado e que podem ou não atuar no tempo presente. O padrão de floração de $P$. suterella foi sazonal, ocorrendo no verão, período de maior pluviosidade e temperatura anual. Em florestas neotropicais úmidas, há espécies de Rubiaceae florescendo em vários momentos do ano. No entanto, é comum a diminuição do percentual de espécies em flor nos meses de menor pluviosidade e temperatura (Opler et al. 1980, Koptur et al. 1988, San

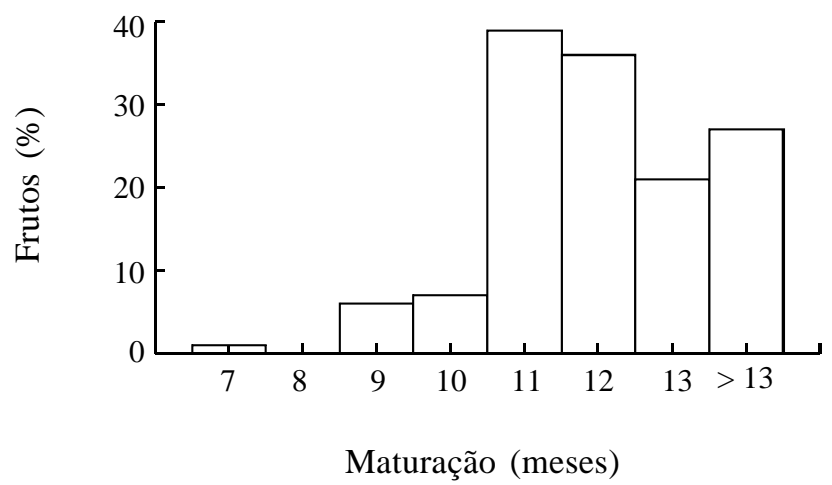

Figura 3. Percentual de frutos de Psychotria suterella que amadureceram ao longo do tempo de observação.

Figure 3. Percentage of Psychotria suterella fruits rippened during the observation period.

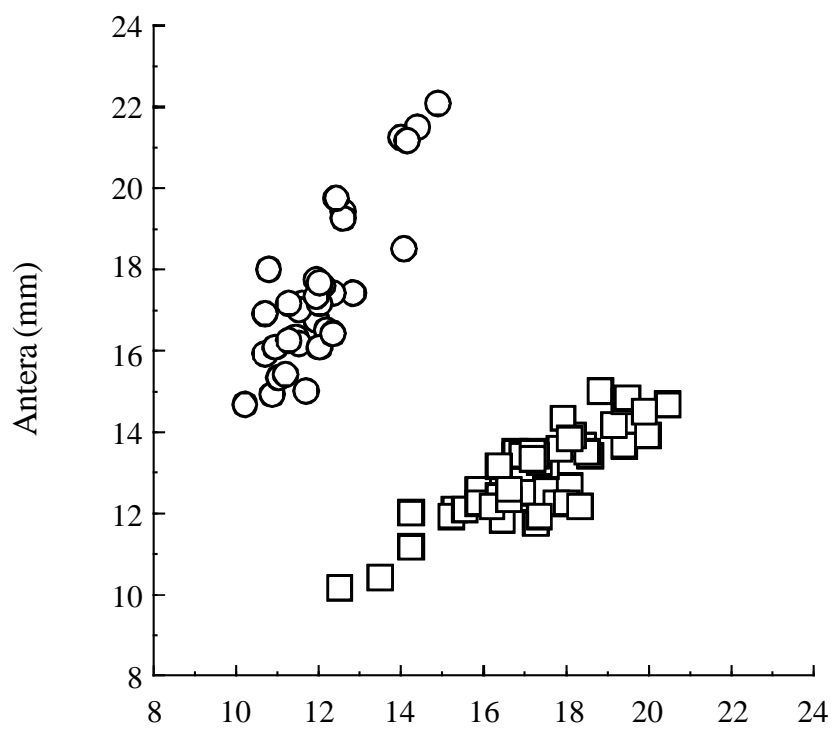

Estigma (mm)

Figura 4. Relação entre altura de anteras e estigmas de Psychotria suterella. Cada ponto representa uma flor do morfo longistilo (quadrado) ou brevistilo (círculo).

Figure 4. Relationship between anthers and stigmas heights of Psychotria suterella. Each dot represents a flower from pin (square) and thrum (circle) morphs.
Martin-Gajardo \& Morellato 2003). A idéia de que a disponibilidade de água pode ter consequiências sobre a fenologia foi reforçada por estudo de Van Schaik e colaboradores (Van Schaik et al. 1993), pois 67\% das regiões com marcada estação seca apresentaram maior floração na estação chuvosa. Concomitantemente, uma vez que estimativas sobre a importância dos visitantes florais indicam que Bombus brasiliensis é responsável por $86 \%$ da polinização de $P$. suterella (Lopes \& Buzato, dados não publicados), consideramos que a disponibilidade desta espécie de polinizador e seu comportamento de forrageio possam exercer, atualmente, pressão seletiva sobre os padrões fenológicos de floração nas populações em estudo. Os dados existentes para Boracéia, SP, indicam que a atividade de forrageio de Bombus brasiliensis é mais intensa de janeiro a março, intermediária em dezembro e abril, e inexistente nos meses de maio a setembro (Wilms 1995). Bombus morio, outra espécie polinizadora de Psychotria suterella (Lopes 2002), apresenta padrão de atividade semelhante, em região próxima da área de estudo, não sendo coletada de julho até outubro e atingindo maior número de indivíduos em março (Knoll 1985). Além disso, espécies do gênero Bombus podem se comportar temporariamente como especialistas de recursos florais disponíveis em maior abundância (Heinrich 1976), sendo registrado que Psychotria suterella é o recurso mais importante para Bombus brasiliensis entre os meses de janeiro a março, uma vez que foi a única espécie em flor visitada por B. brasiliensis em 18 parcelas circulares de $15 \mathrm{~m}$ de raio na Reserva do Morro Grande (Checchia-da-Inês \& Buzato, dados não publicados). Dados semelhantes foram obtidos para esta espécie em Boracéia, SP(Wilms

Tabela 1. Dimensões florais dos morfos brevistilo e longistilo de Psychotria suterella. Média e desvio padrão.

Table 1. Floral measurements for pin and thrum morphs of Psychotria suterella. Mean and standard deviation.

\begin{tabular}{lcc}
\hline Estrutura floral & Brevistilo & Longistilo \\
\hline $\begin{array}{l}\text { Diâmetro da corola } \\
\quad(\mathrm{mm})\end{array}$ & $3,13 \pm 0,06$ & $2,90 \pm 0,05$ \\
$\begin{array}{l}\text { Comprimento da } \\
\quad \text { corola (mm) }\end{array}$ & $13,83 \pm 0,23$ & $13,28 \pm 0,19$ \\
$\begin{array}{l}\text { Altura das anteras } \\
\quad(\mathrm{mm})\end{array}$ & $17,43 \pm 0,30$ & $12,83 \pm 0,17$ \\
$\begin{array}{l}\text { Altura dos estigmas } \\
\quad(\mathrm{mm})\end{array}$ & $12,04 \pm 0,18$ & $16,98 \pm 0,25$ \\
$\begin{array}{l}\text { Diferença Antera- } \\
\text { Estigma (mm) }\end{array}$ & $5,39 \pm 0,18$ & $4,14 \pm 0,15$ \\
& & \\
\hline
\end{tabular}


Tabela 2. Atributos florais de Psychotria suterella. Desenho experimental hierárquico sendo Morfo fator fixo e os demais, fatores aleatórios. $\mathrm{SS}=$ soma de quadrados; $\mathrm{df}=$ graus de liberdade; $\mathrm{MS}=$ quadrados médios; $\mathrm{F}=$ estatística $\mathrm{F}$ (MS de um nível hierárquico/MS do nível inferior); $\mathrm{CV}=$ componente da variância relativa a cada um dos efeitos; $\varpi^{2}(\%)=$ magnitude de efeito: percentual da variação total explicada pelo respectivo fator.

Table 2. Floral features of Psychotria suterella. Hierarchical experimental design with Morph as a fixed factor and the other as random factors. $\mathrm{SS}=$ sum of squares; $\mathrm{df}=$ degrees of freedom; $\mathrm{MS}=$ mean squares; $\mathrm{F}=\mathrm{F}$ statistics (MS of a hierarchical level/MS of the lower level); CV = Variance component; $\varpi^{2}(\%)=$ percentage of variation explained by the factor.

\begin{tabular}{|c|c|c|c|c|c|c|}
\hline Fonte & SS & df & MS & $\mathrm{F}$ & $\mathrm{CV}$ & $\varpi^{2}(\%)$ \\
\hline \multicolumn{7}{|l|}{ Altura das anteras } \\
\hline Morfo & 1476,19 & 1 & 1476,19 & $43,02 * *$ & 5,63 & 67,05 \\
\hline Indivíduos em morfo & 480,31 & 14 & 34,31 & $11,55^{* *}$ & 1,96 & 23,32 \\
\hline Flores em indivíduos & 142,69 & 48 & 2,97 & $33,00^{* *}$ & 0,72 & 8,57 \\
\hline Anteras em flores & 18,07 & 192 & 0,09 & & 0,09 & 1,07 \\
\hline Total & 2117,26 & 255 & & & & \\
\hline \multicolumn{7}{|l|}{ Altura dos estigmas } \\
\hline Morfo & 382,35 & 1 & 382,35 & $80,49^{* *}$ & 5,90 & 73,75 \\
\hline Indivíduos em morfo & 66,52 & 14 & 4,75 & $3,89 * *$ & 0,88 & 11,03 \\
\hline Estigmas em indivíduos & 58,44 & 48 & 1,22 & & 1,22 & 15,25 \\
\hline Total & 507,31 & 63 & & & & \\
\hline \multicolumn{7}{|l|}{ Comprimento da corola } \\
\hline Morfo & 7,74 & 1 & 7,74 & 1,99 & 0,06 & 3,28 \\
\hline Indivíduos em morfo & 54,36 & 14 & 3,88 & $3,59 * *$ & 0,7 & 38,04 \\
\hline Flores em indivíduos & 52,07 & 48 & 1,08 & & 1,08 & 58,70 \\
\hline Total & 114,17 & 63 & & & & \\
\hline \multicolumn{7}{|l|}{ Diâmetro da corola } \\
\hline Morfo & 1,39 & 1 & 1,39 & $6,04 \dagger$ & 0,02 & 2,27 \\
\hline Indivíduos em morfo & 3,17 & 14 & 0,23 & $2,87^{*}$ & 0,70 & 87,19 \\
\hline Flores em indivíduos & 3,63 & 48 & 0,08 & & 0,08 & 10,00 \\
\hline Total & 8,19 & 63 & & & & \\
\hline \multicolumn{7}{|c|}{ Diferença absoluta de altura entre antera e estigma } \\
\hline Morfo & 26,94 & 1 & 26,94 & $10,48 \ddagger$ & 0,38 & 25,90 \\
\hline Indivíduos em morfo & 35,99 & 14 & 2,57 & $4,36^{* *}$ & 0,50 & 33,67 \\
\hline Flores em indivíduos & 28,40 & 48 & 0,59 & & 0,59 & 40,14 \\
\hline Total & 91,33 & 63 & & & & \\
\hline
\end{tabular}

$* \mathrm{P}<0,01 ; * * \mathrm{P}<0,001 ; \dagger \mathrm{P}=0,055 ; \ddagger \mathrm{P}=0,012$

1995). A coincidência do período anual de atividade de Bombus brasiliensis com o período de floração de P. suterella, o fato de B. brasiliensis ser o polinizador mais importante desta espécie, bem como a importância de $P$. suterella na dieta desta abelha, sugerem que pressões seletivas entre as duas espécies podem atuar na determinação de seus períodos de floração e atividade de forrageio, respectivamente. Para uma avaliação mais completa da influência das pressões seletivas geradas por Bombus sobre a fenologia de P. suterella e vice-versa, é necessário o acompanhamento dos padrões populacionais e comportamentais desse polinizador associado à fenologia de floração e ao sucesso reprodutivo dos indivíduos de $P$. suterella, ao longo de suas áreas de distribuição (Thompson 2002).

O padrão de frutificação de $P$. suterella foi sazonal, e a produção de frutos maduros foi mais intensa nos períodos de maior pluviosidade. Frutos verdes ocorreram ao longo de todo ano. À semelhança de outros estudos, verificou-se diferença na porcentagem de Fournier de frutos maduros em relação aos frutos imaturos. No entanto esta diferença não se deve apenas ao resultado da rápida remoção por animais frugívoros associada ao intervalo mensal de observação (San Martin-Gajardo \& Morellato 2003). Este resultado, em P. suterella, pode ser conseqüência do longo período necessário para 
Tabela 3. Produção de frutos resultante dos experimentos de polinização controlada em Psychotria suterella.

Table 3. Fruit set from experimental pollination treatments for Psychotria suterella.

\begin{tabular}{llccc}
\hline Morfo & Tratamento & Flores (n) & Frutos (n) & Frutificação (\%) \\
\hline Brevistilo & Autopolinização & 17 & 0 & 0 \\
& Cruzamento intramorfo & 26 & 0 & 0 \\
& Cruzamento intermorfo & 14 & 7 & 50 \\
\multirow{3}{*}{ Longistilo } & Condições naturais & 29 & 18 & 62 \\
& Autopolinização & 4 & 0 & 0 \\
& Cruzamento intramorfo & 14 & 0 & 0 \\
& Cruzamento intermorfo & 15 & 11 & 73,3 \\
& Condições naturais & 26 & 12 & 46,1 \\
\hline
\end{tabular}

maturação de frutos, aumentando a proporção de frutos imaturos em relação a frutos maduros em um dado momento.

A disponibilidade de frutos maduros ao longo do ano, em $P$. suterella, pode estar relacionada à imprevisibilidade nas condições de luminosidade no subbosque favoráveis à germinação de sementes e ao estabelecimento de plântulas (Rathcke \& Lacey 1985). É possível que plântulas de $P$. suterella necessitem de determinadas condições de luminosidade, como é o caso de plântulas de Psychotria aubletiana Steyerm., cuja performance é melhor em condições de maior luminosidade (Amézquita 1998). Adicionalmente, a dispersão de frutos não seria um fator restritivo para os períodos de frutificação uma vez que o tangará (Chiroxiphia caudata), um dos principais dispersores de sementes de P. suterella, está presente na paisagem em estudo ao longo de todo ano (Boscolo 2002).

Cabe ressaltar que o padrão encontrado na fenologia de floração e frutificação de $P$. suterella nas doze populações estudadas foi semelhante ao encontrado para a população da mata da "Cidade Universitária Armando Sales de Oliveira" na cidade de São Paulo (Grandisoli 1997). Portanto, apesar de alguma variação no mês de início da florada (Grandisoli 1997), P. suterella parece apresentar padrão fenológico consistente na região, independente da paisagem.

Morfologia floral - A posição de anteras e estigmas, melhor explicada pelo fator morfo, permite considerar a espécie distílica, havendo a distinção de indivíduos pela ocorrência de dois tipos florais. Por outro lado, a diferença absoluta de altura entre antera e estigma e comprimento da corola é mais bem explicada pelo fator flores em indivíduo, indicando a ocorrência de variações intraindividuais em P. suterella. Comparações da variação na posição de anteras e estigmas dentro da espécie podem ser úteis para verificar a ação de forças seletivas sobre as estruturas reprodutivas. Observações de que em espécies alógamas, variações nos atributos florais têm influência maior no sucesso reprodutivo do componente masculino que na produção de frutos e sementes, sugerem que seleção natural agindo sobre a posição de anteras pode ter prioridade evolutiva sobre seleção na posição de estigma (Barrett et al. 1998). De acordo com esta hipótese, poderíamos esperar menor variação na posição das anteras, como resultado de maior seleção estabilizadora. Nossos dados não reforçam esta idéia, visto que as variações inter-individuais na posição de estigmas e anteras são semelhantes, indicando que as pressões seletivas atuam de modo semelhante nas funções masculina e feminina em $P$. suterella. No entanto, a questão necessita de abordagem experimental a fim de verificar como a variação nos atributos florais influencia a aptidão das funções masculina e feminina. Cabe ressaltar também que há evidências de que alterações em atributos florais podem exercer pressão seletiva apenas sobre a função feminina (Johnson \& Steiner 1997). O elevado grau de reciprocidade entre os morfos na posição de estigmas e anteras, em comparação com outras espécies heterostílicas (Richards \& Koptur 1993, Pailler \& Thompson 1997), permite considerar a espécie estudada como um caso de hercogamia recíproca com potencial para a ocorrência de dispersão direcional de pólen (Stone 1995, Ree 1997). Observações não sistematizadas das flores de $P$. suterella nas diversas populações indicam que a diferenciação morfológica entre indivíduos brevistilos e longistilos mantém-se independente da fragmentação de hábitat na região de estudo.

Sistema reprodutivo - Psychotria suterella apresentou mecanismo de incompatibilidade heteromórfico total, não havendo formação de frutos perante a polinização intra- 
morfos. Este tipo de incompatibilidade faz com que o sistema seja dependente de vetores para a formação de frutos e sementes. Ao contrário, Grandisoli (1997) registrou frutificação a partir de auto-polinização espontânea, auto-polinização manual, bem como polinização intra-morfos, em $P$. suterella. Tais resultados podem indicar quebra do mecanismo de incompatibilidade intra-morfos no fragmento florestal estudado por aquele autor (10 ha), situado na cidade de São Paulo (Grandisoli 1997). A quebra do mecanismo de incompatibilidade heteromórfica tem sido registrada em outros sistemas, sendo associada a vários fatores tais como ausência de polinizadores (Alves-dos-Santos 2002), desvios na proporção de morfos (Ganders 1979) e distribuição geográfica (Sobrevila et al. 1983).

Frutificação - A grande variação entre as áreas de estudo quanto à frutificação de $P$. suterella em condições naturais não está associada a variações na atividade de polinizadores, não havendo limitação polínica (Lopes 2002). Visto que fatores abióticos atuam sobre o sucesso reprodutivo de espécies vegetais (Sutherland 1986) e que fragmentação de hábitat pode alterar fatores abióticos (Saunders et al. 1991), considera-se que a heterogeneidade nas condições abióticas dos fragmentos poderiam estar condicionando as variações observadas na frutificação (Lopes 2002). Outras espécies do gênero (Castro 2001), tais como P. jasminoides Standl. (47,6\%), $P$. birotula L.B. Smith \& Downs $(15,65 \%)$, P. mapourioides DC. $(26,65 \%)$ e P. nuda (Cham. \& Schltdl.) Wawra $(54,1 \%)$ apresentam frutificação média semelhante à de $P$. suterella. No entanto, a frutificação observada é baixa quando comparada aos $85,56 \%$ de frutificação registrados para $P$. suterella em outra área (Grandisoli 1997). Pode-se supor que esta diferença esteja relacionada ao menor nível de incompatibilidade intra-morfos na população estudada por Grandisoli (1997), como sugerido para Palicourea petiolaris (Knuth) Spreng. (Sobrevila et al. 1983). No entanto, esta justificativa implica em baixa frutificação por limitação polínica nas populações, hipótese esta já descartada para as populações em estudo (Lopes 2002). Uma hipótese a ser verificada é a de que tais diferenças na frutificação poderiam estar associadas às características abióticas de cada área na qual $P$. suterella ocorre.

Agradecimentos - Às amigas M.R. Darrigo e S. Del-Carlo, pelo valioso auxílio no trabalho de campo. A E.L. Catharino e G.A.D.C. Franco pela identificação de P. suterella. AJ.R. Nali, à Sabesp e aos proprietários dos fragmentos florestais, pela permissão de acesso às áreas de estudo. A R.M. Shimizu pelas soluções gráficas. A J.P. Metzger pelo apoio e oportunidade de trabalhar no projeto "Conservação da biodiversidade em paisagens fragmentadas no planalto Atlântico de São Paulo". Este manuscrito é parte da Dissertação de Mestrado do primeiro autor, vinculada ao Programa de Pós-Graduação em Ecologia, Departamento de Ecologia, Instituto de Biociências, Universidade de São Paulo. À Fapesp pelos auxílios financeiros (99/12704-3 e 99/05123-4).

\section{Referências bibliográficas}

AKER, C.L. 1982. Spatial and temporal dispersion patterns of pollinators and their relationship to the flowering strategy of Yucca whipplei. Oecologia 54:243-252.

ALMEIDA, E.M. \& ALVES, M.A. 2000. Fenologia de Psychotria nuda e P. brasiliensis (Rubiaceae) em uma área de Floresta Atlântica no sudeste do Brasil. Acta Botanica Brasilica 14:335-346.

ALVES-DOS-SANTOS, I. 2002. Flower-visiting bees and the breakdown of the tristylous breeding system of Eichhornia azurea (Swartz) Kunth (Pontederidaceae). Biological Journal of the Linnean Society 77:499-507.

AMÉZQUITA, P. 1998. Light environment affects seedling performance in Psychotria aubletiana (Rubiaceae), a tropical understory shrub. Biotropica 30:126-129.

AUGSPURGER, C.K. 1983. Phenology, flowering synchrony, and fruit-set of 6 neotropical shrubs. Biotropica 15:257-267.

BARRETT, S.C.H. \& RICHARDS, J.H. 1990. Heterostyly in tropical plants. Memoirs of the New York Botanical Garden 55:35-61.

BARRETT, S.C.H., COLE, W.W. \& HARDER, L.D. 1998. Experimental studies on the effects of floral design and display on plant mating. In Reproductive biology (S.J. Owens \& P.J. Rudall, eds.). Royal Botanical Gardens, Kew, p.1-13.

BAWA, K.S. \& BEACH, J.H. 1983. Self-incompatibility systems in the Rubiaceae of a tropical lowland wet forest. American Journal of Botany 70:1281-1288.

BENCKE, C.S.C. \& MORELLATO, L.P.C. 2002. Comparação de dois métodos de avaliação da fenologia de plantas, sua interpretação e representação. Revista Brasileira de Botânica 25:269-275.

BOSCOLO, D. 2002. O uso de técnicas de play-back no desenvolvimento de um método capaz de atestar a presença ou ausência de aves no interior de fragmentos florestais. Dissertação de mestrado, Universidade de São Paulo, São Paulo.

BOLMGREN, K. 1998. The use of synchronization measures in studies of plant reproductive phenology. Oikos 82:411-415.

BRONSTEIN, J.L. 1995. The plant-pollinator landscape. In Mosaic landscapes and ecological processes (L. Hansson, L. Fahrig \& G. Merriam, eds.). Chapman \& Hall, London, p. 256-288. 
BUZATO, S., SAZIMA, M. \& SAZIMA, I. 1994. Pollination of three species of Abutilon (Malvaceae) intermediate between bat and hummingbird flower syndromes. Flora 189:327-334.

CARVALHO, L.M.T., FONTES, M.A.L. \& OLIVEIRAFILHO, A.T. 2000. Tree species distribution in canopy gaps and mature forest in an area of cloud forest of the Ibitipoca range, south-eastern Brazil. Plant Ecology 149:9-22.

CASTRO, C.C. 2001. Biologia reprodutiva de rubiáceas arbustivas de Mata Atlântica. Tese de doutorado, Universidade Estadual de Campinas, Campinas.

CASTRO, C.C. \& OLIVEIRA, P.E.A.M. 2001. Reproductive biology of the protandrous Ferdinandusa speciosa Pohl (Rubiaceae) in southeastern Brazil. Revista Brasileira de Botânica 24:167-172.

CASTRO, C.C. \& OLIVEIRA, P.E. 2002. Pollination biology of distylous Rubiaceae in the Atlantic Rain Forest, SE Brazil. Plant Biology 4:640-646.

COELHO, C.P. \& BARBOSA, A.A.A. 2003. Biologia reprodutiva de Palicourea macrobotrys Ruiz \& Pavon (Rubiaceae): um possível caso de homostilia no gênero Palicourea Aubl. Revista Brasileira de Botânica 26:403-413.

DEAN, W. 1996. A ferro e fogo - A história e a devastação da Mata Atlântica brasileira. Companhia das Letras, São Paulo.

FEINSINGER, P. \& BUSBY, W.H. 1987. Pollen carryover: experimental comparisons between morphs of Palicourea lasiorrachis (Rubiaceae), a distylous, birdpollinated, tropical treelet. Oecologia 73:231-235.

FOURNIER, L.A. 1974. Um método cuantitativo para la medición de características fenológicas em árboles. Turrialba 21:422-423.

FUCHS, E.J., LOBO, J.A. \& QUESADA, M. 2003. Effects of forest fragmentation and flowering phenology on the reproductive success and mating patterns of the tropical dry forest tree Pachira quinata. Conservation Biology 17:149-157.

GANDERS, F.R. 1979. The biology of heterostyly. New Zealand Journal of Botany 17:607-635.

GENTRY,A.H. \& EMMONS, L.H. 1987. Geographical variation in fertility, phenology, and composition of the understory of neotropical forests. Biotropica 19:216-227.

GÓMEZ, J.M. 1993. Phenotypic selection on flowering synchrony in a high mountain plant Hormatophylla spinosa (Cruciferae). Journal of Ecology 81:605-613.

GRAHAM, M.H. \& EDWARDS, M.S. 2001. Statistical significance versus fit: estimating the importance of individual factors in ecological analysis of variance. Oikos 93:505-513.

GRANDISOLI, E.A.C. 1997. Biologia reprodutiva e estrutura da população de Psychotria suterella Muell. Arg. (Rubiaceae) em um fragmento de mata secundária em São Paulo (SP). Dissertação de mestrado, Universidade de São Paulo, São Paulo.
GRANT, K.A. \& GRANT, V. 1967. Effects of hummingbird migration on plant speciation in the California flora. Evolution 21:457-465.

HEINRICH, B. 1976. The foraging specializations of individual bumblebees. Ecological Monographs 46:105-128.

JOHNSON, S.D. \& STEINER K.E. 1997. Long-tongued fly pollination and evolution of floral spur length in the Disa draconis complex (Orchidaceae). Evolution 51:45-53.

KNOLL, F.R.N. 1985. Abundância relativa das abelhas no campus da Universidade de São Paulo, com especial referência à Tetragonisca angustula Latreille. Dissertação de mestrado, Universidade de São Paulo, São Paulo.

KÖPPEN, W. 1948. Climatología con un estudio de los climas de la tierra. Ed. Fondo Cultura Económica, Ciudad de México.

KOPTUR, S., HABER, W.A., FRANKIE, G.W. \& BAKER, H.G. 1988. Phenological studies of shrub and treelet species in tropical cloud forest of Costa Rica. Journal of Tropical Ecology 4:323-346.

KUNIN, W. \& IWASA, Y. 1996. Pollinator foraging strategies in mixed floral arrays: density effects and floral constancy. Theoretical Population Biology 49:232-263.

LAU, P. \& BOSQUE, C. 2003. Pollen flow in the distylous Palicourea fendlery (Rubiaceae): an experimental test of the disassortative pollen flow hypothesis. Oecologia 135:593-600.

LAURANCE, W.F., LOVEJOY, T.E., VASCONCELOS, H.L., BRUNA, E.M., DIDHAM, R.K., STOUFFER, P.C., GASCON, C., BIERREGAARD, R.O., LAURANCE, S., \& SAMPAIO, E. 2001. Ecosystem decay of amazonian forest fragments: a 22-year investigation. Conservation Biology 16:605-618.

LOPES, L.E. 2002. Biologia reprodutiva de Psychotria suterella (Rubiaceae): efeitos da fragmentação de hábitat e de conexão estrutural. Dissertação de mestrado, Universidade de São Paulo, São Paulo.

MACHADO, I.C. \& LOIOLA, M.I. 2000. Fly pollination and pollinator sharing in two synchronopatric species: Cordia multispicata (Boraginaceae) and Borreria alata (Rubiaceae). Revista Brasileira de Botânica 23:305-311.

MARQUIS, R.J. 1988. Phenological variations in the neotropical understory schrub Piper arieianum: causes and consequences. Ecology 69:1552-1565.

MELO, C., BENTO, E.C. \& OLIVEIRA, P.E. 2003. Frugivory and dispersal of Faramea cyanea (Rubiaceae) in Cerrado woody plant formations. Brazilian Journal of Biology 63:75-82.

MORELLATO, L.P.C., TALLORA, D.C., TAKAHASI, A., BENCKE, C.C., ROMERA, E. \& ZIPARRO, V.B. 2000. Phenology of atlantic rain forest trees: a comparative study. Biotropica 32:811-823. 
NEWSTROM, L.E., FRANKIE, G.W. \& BAKER, H.G. 1994. A new classification for plant phenology based on flowering patterns in lowland tropical rain forest trees at La Selva, Costa Rica. Biotropica 26:141-159.

OLIVEIRA FILHO, A.T. \& FONTES, M.A.L. 2000. Patterns of floristic differentiation among atlantic forests in southeastern Brazil and influence of climate. Biotropica 32:793-810.

OPLER, P.A., FRANKIE, G.W. \& BAKER, H.G. 1980. Comparative phenological studies of treelet and shrub species in tropical wet and dry forests in the lowlands of Costa Rica. Journal of Ecology 68:167-188.

PAILLER, T. \& THOMPSON, J.D. 1997. Distyly and variation in heteromorphic incompatibility in Gaertnera vaginata (Rubiaceae) endemic to La Reunion Island. American Journal of Botany 84:315-327.

PASSOS, L. \& SAZIMA, M. 1995. Reproductive biology of the distylous Manetia luteo-rubra (Rubiaceae). Botanica Acta 108:277-402.

POULIN, B., WRIGHT, S.J., LEFEBVRE, G. \& CALDERÓN, O. 1999. Interspecific synchrony and asynchrony in the fruiting phenologies of congeneric bird-dispersed plants in Panama. Journal of Tropical Ecology 15:213-227.

RATHCKE, B.J. 1992. Nectar distributions, pollinator behavior, and plant reproductive success. In Effects of resource distribution on animal-plant interactions (M.D. Hunter, T. Ohgushi \& P.W. Price, eds.). Academic Press, San Diego, p.113-138.

RATHCKE, B. \& LACEY, E.P. 1985. Phenological patterns of terrestrial plants. Annual Review of Ecology and Systematiscs 16:179-214.

REE, R. 1997. Pollen flow, fecundity and the adaptive significance of heterostyly in Palicourea padifolia (Rubiaceae). Biotropica 29:298-308.

RICHARDS, J.H. \& KOPTUR, S. 1993. Floral variation and distyly in Guettarda scabra (Rubiaceae). American Journal of Botany 80:31-40.

SABESP 1997. Programa de conservação do Sistema Cotia. Relatório Conclusivo (tomo 3): Avaliação Ambiental. Sabesp/Fundação Brasileira para o Desenvolvimento Sustentável, São Paulo.

SAN MARTIN-GAJARDO, I. \& MORELLATO, L.P.C. 2003. Fenologia de Rubiaceae do sub-bosque em floresta Atlântica no sudeste do Brasil. Revista Brasileira de Botânica 26:299-309.
SAUNDERS JUNIOR, D.A., HOBBS, R.J. \& MARGULES, C.R. 1991. Biological consequences of ecosystem fragmentation: a review. Conservation Biology 5:18-32.

SEABRA, M. 1971. Vargem Grande: organização e transformações de um setor do cinturão verde paulistano. Universidade de São Paulo, São Paulo.

SOBREVILA, C., RAMIREZ, N. \& ENRECH, N.X. 1983. Reproductive biology of Palicourea fendleri and P. petiolaris (Rubiaceae), heterostylous shrubs of a tropical cloud forest in Venezuela. Biotropica 15:161-169.

STONE, J. 1995. Pollen donation patterns in a tropical dystilous shrub (Psychotria surrensis; Rubiaceae). American Journal of Botany 82:1390-1398.

SUTHERLAND, S. 1986. Patterns of fruit-set: what controls fruit-flower ratio in plants? Evolution 40:117-128.

TSCHAPKA, M. 2004. Energy density patterns of nectar resources permit coexistence within a guild of neotropical flower-visiting bats. Journal of Zoology 263:7-21.

THOMPSON, J.N. 2002. Plant-animal interactions: future directions. In Plant-animal interactions - an evolutionary approach (C.M. Herrera \& O. Pellmyr, eds.). Blackwell Science, Oxford, pp.236-247.

UEZU, A., METZGER, J.P. \& VIELLIARD, J.M.E. 2005. Effects of structural and functional connectivity and patch size on the abundance of seven atlantic forest bird species. Biological Conservation 123:507-519.

VAN SCHAIK, C.P., TERBORGH, J.W. \& WRIGHT, S.J. 1993. The phenology of tropical forests: adaptive significance and consequences for primary consumers. Annual Review of Ecology and Systematics 24:353-377.

WILMS, W. 1995. Die Bienenfauna im Küstenregenwald Brasiliens und ihre Beziehungen zu Blütenplanzen: Fallstudie Boracéia, São Paulo. Dissertation zur Erlangung des Grades eines Doktors der Naturwissenschaften, Eberhard-Karls-Universität Tübingen, Tübingen.

WRIGHT, S.J. 1991. Seasonal drought and the phenology of understory shrubs in a tropical moist forest. Ecology 72:1643-1657.

ZAR, J.H. 1999. Biostatistical analysis. Prentice Hall, New Jersey. 